DOI https://doi.org/10.15589/znp2019.1(475).16

УДК 004.932.2; 004.932.2

\title{
APPROACHES TO IDENTIFICATION OF FRAGMENTS OF A DISLOCATION ETCH PIT ON A SEMICONDUCTOR MONOCRYSTAL WAFFER
}

\section{ПОДХОДЫ К ИДЕНТИФИКАЦИИ ФРАГМЕНТОВ КОНТУРА ДИСЛОКАЦИИ НА ПЛАСТИНЕ МОНОКРИСТАЛЛА ПОЛУПРОВОДНИКА}

\author{
Andrii M. Samoilov \\ saman.39@gmail.com \\ ORCID: 0000-0001-9178-6202 \\ Igor V. Shevchenko \\ ius.shevchenko@gmail.com \\ ORCID: 0000-0003-3009-8611
}

\author{
А. Н. Самойлов \\ аспирант
}

И. В. Шевченко

докт. техн. наук, профессор

Kremenchuk Mykhailo Ostohradskyi National University, Kremenchuk

Кременчугский наџиональный университет имени Михаила Остроградского, г. Кременчуг

\begin{abstract}
The given work is devoted to approaches to using the features of identification of fragments of a dislocation circuit on a digitally binarized image of a surface of a single crystal of a semiconductor. The plate after selective etching, using light microscopy and photo-optical method of forming the observation areas, after processing several types of binarization of the image of the surface of the semiconductor plate, is evaluated in order to generate the identification features. The approach proposed in the paper involves consideration of the parameters that represent the characteristics of fragments considered in the form of binarized images of the surface of the plate obtained in parallel, as a result of adaptive and global binarization. The refinement of the subset of the main significant fragments of the contours of the dislocations is invariant under the following characteristics: produced after the preprocessing of the obtained image, which includes converting a color bitmap into an eight-tone eight-bit image, improving image clarity; global binarization; obtaining contours on digital raster images with fuzzy display of dislocations on GaAs wafers; detection of contours in the differences in the brightness of the predicted contour line, rebuilding of the contour line of dislocation of the digital image of the wafers, selection of binarized fragments of the etch pit. Invariant features that affect the result of grouping and identification process situations: the coincidence of binary and major significant fragments; match the characteristics of the contour line with the reference; closed line contours; the coordinates of the significant fragments and the main significant fragments. The clustering approach is a variant of the nearest neighbor method and has automatic parameter tuning. As a result of the identification, the plurality of significant fragments from the point of view of the etch pits wells belonging to the fragments is grouped into the identified contours of the etching pits of the semiconductor wafers. The novelty of this approach allows to solve the problem of automation of the process of calculating the dislocation density on the image of the surface of a semiconductor single crystal, which is an urgent problem for reducing the labor costs and practical increase in the efficiency of obtaining indicators of the quality of semiconductor single crystals.
\end{abstract}

Key words: global binarization; adaptive binarization; raster image, fragment; dislocation; etch pit; waffer GaAs.

Анотація. У статті досліджуються підходи щодо використання ознак ідентифікації фрагментів контуру дислокації на цифровому бінаризованому зображенні поверхні монокристала напівпровідника. Пластина після селективного травлення, за допомогою світлової мікроскопії та фотооптичного методу формування зон спостереження, після обробки декількома видами бінаризації зображення поверхні пластини напівпровідника оцінюється з метою формування ознак ідентифікації. Підхід, запропонований у роботі, включає розгляд параметрів, що являють собою характеристики фрагментів, розглянутих у вигляді бінаризованих зображень поверхні пластини, отриманих паралельно, як результат роботи адаптивної та глобальної бінаризації. Уточнення підмножини основних значущих фрагментів контурів дислокацій проходить за інваріантними ознаками, що виробляються після: попередньої обробки одержуваного зображення, що включає перетворення кольорового растрового зображення на півтонове восьмибітне зображення, підвищення чіткості зображення; глобальної бинаризації; отримання контурів на цифрових растрових зображеннях з нечітким відображенням дислокацій на пластинах GaAs; виявлення контурів у перепадах яскравості передбачуваних граней контуру, відновлення лінії контуру дислокації цифрового зображення пластини, виділення бінаризованих фрагментів ямок травлення. 
Інваріантні ознаки, що впливають на результат групування та ідентифікації, опрацьовують ситуації: співпадіння бінарних і основних значущих фрагментів; збіги характеристик лінії контуру з еталонними; замкнутості лінії контуру; збіги координат значущих фрагментів і основних значущих фрагментів. Підхід щодо групування $\epsilon$ різновидом методу найближчих сусідів та має автоматичне налагодження параметрів. Як результат ідентифікації - множина значимих фрагментів з точки зору приналежних до фрагментів ямки травлення групується до ідентифікованих контурів ямок травлення пластин напівпровідників. Новизна такого підходу дозволяє вирішувати завдання автоматизації процесу підрахунку щільності дислокацій на зображенні поверхні пластини монокристала напівпровідника, що є актуальною проблемою для зменшення трудовитрат та практичного підвищення оперативності отримання показників якості пластин монокристалів напівпровідника.

Ключові слова: ідентифікація; фрагмент; цифрове зображення; контур дислокації; пластина монокристала напівпровідника; ямка травлення.

Аннотация. В статье исследуются подходы по использованию признаков идентификации фрагментов контура дислокации на цифровом бинаризированном изображении поверхности монокристалла полупроводника. После световой микроскопии и фотооптического формирования зон наблюдении используются характеристики фрагментов бинаризированных изображений. Уточнение подмножества основных значимых фрагментов контуров достигается по инвариантным признакам: совпадение бинарных и основных значимых фрагментов; совпадение характеристик линии контура с эталонными; замкнутость линии контура; совпадение координат значимых фрагментов и основных значимых фрагментов. Подход к группировке метода ближайших соседей имеет автоматическая настройка параметров. Множество значимых фрагментов с точки зрения принадлежащих к фрагментам ямки травления группируется в идентифицированных контурах ямок травления. Новизна подхода решит задачи автоматизации процесса подсчета плотности дислокаций пластины монокристалла полупроводника, уменьшит трудозатраты и повысит оперативность получения показателей качества пластин монокристаллов полупроводника.

Ключевые слова: идентификация; фрагмент; цифровое изображение; контур дислокации; пластина монокристалла полупроводника; ямка травления.

\section{ПОСТАНОВКА ЗАДАЧИ}

В процессе производства монокристалла полупроводника GaAs его качество, определяемое величиной плотности дислокацій, нормируется стандартом по величине $10^{3} \div 10^{5} \mathrm{~cm}^{-2}[1]$. Визуально-оптический метод контроля вышеупомянутой величины на относительно недорогом интерферометре МИИ-4, широко используемом операторами заводских лабораторий, предусматривает, по сути, получение числа дислокационных ямок травления, а также их соотношения к площади пластины монокристалла на квадратный сантиметр [1].

Стандарт [1] описывает методику определения дислокаций для кремния. В то же время для измерения плотности дислокаций в подложках GaAs диаметром больше 100 мм, с толщиной 600 мкм и более этап подготовки и травления пластины имеет свои особенности и требует обновленных подходов к процессу измерения величины плотности дислокаций.

Исследования в данной области направлены на усовершенствование точности оценивания дефектов, возникающих в процессе производства полупроводниковых структур. Используемые мировые стандарты [2] предполагают использование точных результатов измерений количества дислокаций.

Поэтому актуален поиск новых путей повышения точности измерений с использованием обновленных подходов визуально-оптического метода контроля на относительно недорогом оборудовании.

\section{АНАЛИЗ ПОСЛЕДНИХ ИССЛЕДОВАНИЙ И ПУБЛИКАЦИЙ}

Рост промышленного производства пластин с диаметром больше 100 мм инициирует поиск способов повышения количества и качества экспрессных и цеховых измерений качества монокристаллов полупроводника.

В работе [3] приводятся способы точной оценки размеров топографических дефектов полупроводниковых кремниевых структур с использованием оптических приборов, а также методика их коррекции применительно к моноисточнику освещения препарата в интерферометре МИИ-4.

В работе [4] усовершенствована методика контроля плотности дислокаций в подложках GaAs. Однако, в отличие от стандартной схемы измерений с 9 полями [1], показанной на рисунке 1 a), предложенный план измерения повышает точность определения плотности дислокаций.

\section{ВЫДЕЛЕНИЕ НЕ РЕШЕННЫХ РАНЕЕ ЧАСТЕЙ ОБЩЕЙ ПРОБЛЕМЫ}

Методика с планом измерения, приведенном на рис. 1 б), предполагает количество полей стандартной схемы и значительно увеличивает трудозатраты и время подсчета показателей плотности дислокаций по стандарту визуально-оптического метода в неавтоматическом режиме (рис. 1 б). 


\section{АВТОМАТИЗАЦІЯ ТА КОМП'ЮТЕРНО-ІНТЕГРОВАНІ ТЕХНОЛОГІЇ № 1 г 2019}
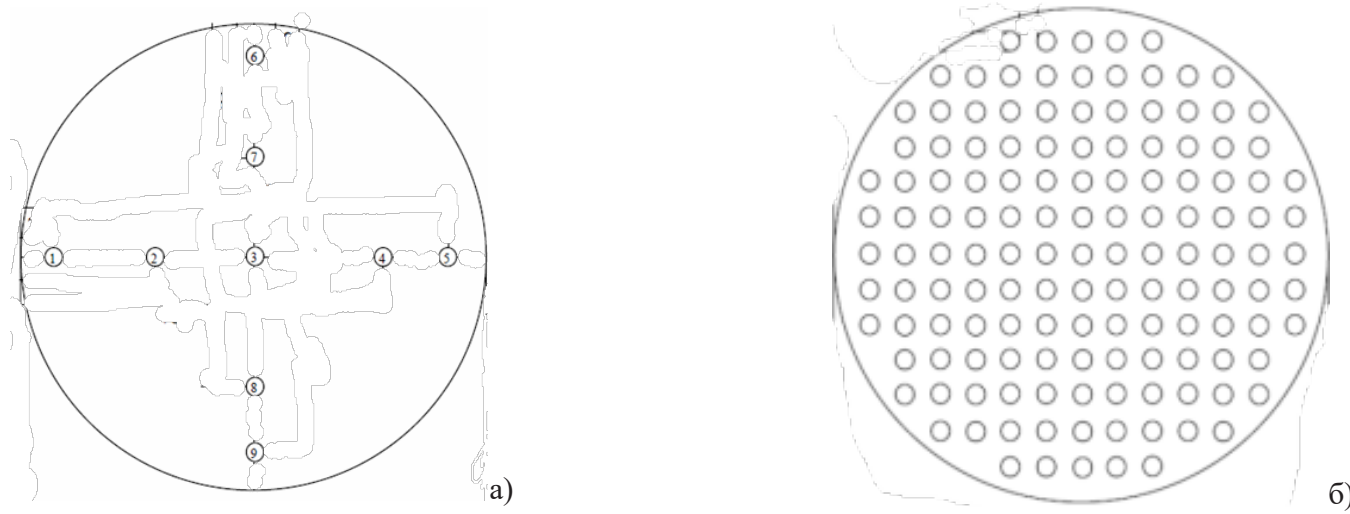

Рис. 1. Схема выбора полей измерения для подсчета неавтоматизированным методом: а) по стандартной схеме выбора полей измерения с 9 полями; б) по усовершенствованной схеме выбора с 137 полями, для подложки диаметром 100 мм для пластин $\{100\}$ и $\{110\}$

Поэтому автоматизация процесса подсчета плотности дислокаций по усовершенствованной схеме выбора полей измерения является актуальной задачей для получения показателей качества пластин монокристаллов полупроводника.

\section{ЦЕЛЬ ИССЛЕДОВАНИЙ}

Необходимо выработать подходы к идентификации фрагментов ямок травления контура дислокации на изображении поверхности пластины монокристалла полупроводника с целью дальнейшего использования для расчета плотности дислокаций.

\section{МЕТОДЫ, ОБЪЕКТ И ПРЕДМЕТ ИССЛЕДОВАНИЯ}

Исследования базировались на методах: селективного травления и световой микроскопии для контроля плотности дислокаций полуизолирующего GaAs и фотооптического метода получения начальных цифровых изображений поверхности пластины монокристалла полупроводника.

Объектом исследований является процесс автоматизированной идентификации фрагментов контура дислокации на пластине монокристалла полупроводника.

Предметом исследований является идентификация дислокаций на пластинах монокристалла полупроводника с использованием обновленных подходов визуально-оптического метода контроля.

Настоящая статья является обобщением результатов, полученных в ходе исследований, с целью разработки методов подсчета дислокаций для автоматизированного вычисления величины плотности дислокаций на пластине монокристалла полупроводника. Результаты этих исследований раскрыты в работах $[1 ; 2 ; 3 ; 4 ; 5 ; 6 ; 8]$.

\section{ОСНОВНОЙ МАТЕРИАЛ}

Искажения изображений фрагментов ямок травления, становящиеся преградой для идентификации, вызваны нерегулярностью бинаризированного изображения поверхности пластины.

Пиксели со значением интенсивности 0 определяют количество точек плоскости фрагментов на бинарном изображении при размере дислокаций, полученных визуально-оптическим методом 5 мкм, показанных на рисунке 2 .

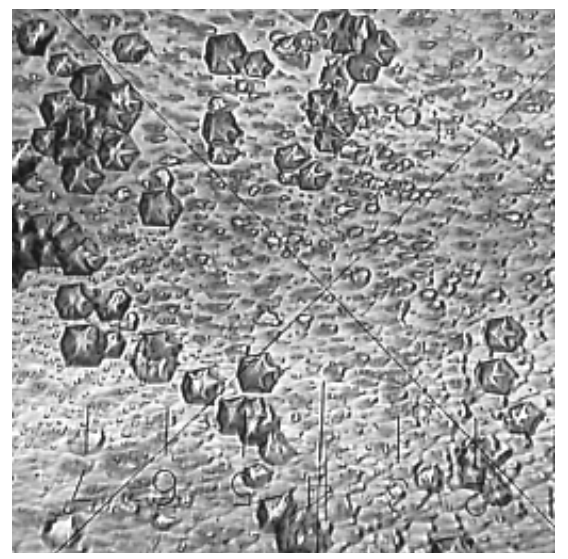

Рис. 2. Предварительно обработанное полутоновое восьмибитное изображение поверхности пластины монокристалла GaAs ориентации [100] с ямками травления дислокаций

Фрагменты на изображении поверхности пластины монокристалла J1 входят в множество $A$ и включают $B$ - значимое подмножество фрагментов, определяющих контуры ямок травления на изображении, а также остальные фрагменты подмножества $C$, не вошедшие в подмножество $B$. Таким образом, смежность незначимых фрагментов $C$ со значимыми фрагментами описывается как:

$$
A=(B \cup C),
$$

где $A$ - множество всех фрагментов бинаризированного изображения, подмножество $B$ - определяет 
значимую часть фрагментов, определяющих составляющие линии контуров, и принадлежат контурной (внешней) или внутренней части ямки травления, $C-$ подмножество незначимых фрагментов, не вошедших в подмножество $B$ [5].

Изображение поверхности пластины монокристалла J0, полученное после обработки глобальной бинаризацией и медианным фильтром, позволяет оценить расположение ямок травления по площади отражения светового пучка освещения интерферометра МИИ-4.

Использование изображения поверхности пластины монокристалла J2 с подчеркнутыми контурами после применения адаптивной бинаризации с наиболее эффективным коэффициентом $C=15$ [6] используется для подтверждения принадлежности фрагментов к подмножеству основных значимых $B$.

Вышеописанные изображения J1 и J2 обработаны медианным фильтром для устранения шумов [7].

Значимое подмножество фрагментов $B$, определяющее контуры ямок травления, включает микродефекты на поверхности пластины подмножества $D$ с координатами центра массы $X_{M D}$ и $Y_{M D}$, где $d=\left\{X_{M D}, Y_{M D}\right\} \in D$, видимых на изображении J3, peзультате сложения J1 и J2 [5]. Критерием принадлежности является совпадение координат центров масс фрагментов подмножества $D$, где $d=\left\{X_{m}, Y_{k}\right\} \in D$ изображения J3 и множества координат J1 и J0: $J 3 \cap J 1=1$ или $\mathrm{J} 0 \quad J 3 \cap J 0=1$ :

$$
X_{M}=X_{M D} \quad Y_{M}=Y_{M D},
$$

где $X_{M}, Y_{M}$ - координаты центров масс изображения $\mathrm{J} 1, X_{M D}, Y_{M D}-$ множество координат центров масс микродефектов изображения J3.

Инвариантные признаки для идентификации основных значимых фрагментов контуров дислокаций включают:

1. Признак совпадения бинарных и основных значимых фрагментов. Если координаты центров масс $X_{M J 2}, Y_{M J 2}$ фрагментов изображения Ј2 совпадают с любой координатой $X_{m J 0}, Y_{n j}$ изображения J0 и принадлежат множеству значимых фрагментов $b=\left\{X_{m}, Y_{k}\right\} \in B$.

2. Признак совпадения характеристик линии контура с эталонными, полученными из множества значимых фрагментов изображения J1.

3. Признак замкнутости линии контура.

4. Признак совпадения координат центров масс значимых фрагментов и основных значимых фрагментов. Если координаты центров масс $X_{M J 1}, Y_{M J 1}$ фрагментов изображения J1 попадают в интервалы координат, соответствующих группировке фрагментов изображения J2, и принадлежат множеству значимых фрагментов $b=\left\{X_{m}, Y_{k}\right\} \in B$.

Признак совпадения координат значимых фрагментов и основных значимых фрагментов соответ- ствия координат фрагментов ямки травления с уникальным идентификатором группы после использования метода группировки [8] имеет следующие этапы определения:

1. Извлечение уникального значения идентификатора группировки.

2. Выборка координат центров масс $X_{M J 2}, Y_{M J 2}$ фрагментов изображения J2 для уникального идентификатора группировки.

3. Выбор определение и фиксация граничных координат центров масс $X_{M J 2 \min }, Y_{M J 2 \min }, X_{M J 2 \max }$, $Y_{M J 2 \max }$ для очередного уникального идентификатора группировки на изображениях Ј1 и Ј2 соответственно.

4. Выборка координат центров масс $X_{M J 1}, Y_{M J 1}$ фрагментов изображения J1.

5. Получение значения признака совпадения координат значимых фрагментов и основных значимых фрагментов, после выполнения условия ограничения координат центров масс $X_{M J 1}, Y_{M J 1}$ фрагментов для изображения J1:

$$
\begin{gathered}
\left(X_{M J 2 \min } \leq X_{M J 1} \leq X_{M J 2 \max }\right) \operatorname{AND}\left(Y_{M J 2 \min } \leq Y_{M J 1} \leq\right. \\
\left.Y_{M J 2 \max }\right)
\end{gathered}
$$

6. Фиксация координат центров масс $X_{M J 1}, Y_{M J 1}$ с признаком совпадения координат значимых фрагментов и основных значимых фрагментов для идентификатора группировки на изображении J1.

В таблице 1 представлена матрица получения результата идентификации изображения ямки травления дислокаций по признакам в столбцах:

1. Совпадение бинарных и основных значимых фрагментов.

2. Совпадение характеристик линии контура с эталонными.

3. Замкнутость линии контура.

4. Совпадение координат центров масс значимых фрагментов и основных значимых фрагментов.

Таблица 1. Матрица идентификации контура дислокации по фрагментам ямки травления

\begin{tabular}{|c|c|c|c|c|c|}
\hline № п. п. & $\mathbf{1}$ & $\mathbf{2}$ & $\mathbf{3}$ & $\mathbf{4}$ & Результат \\
\hline 1 & 1 & 1 & 0 & 1 & 1 \\
\hline 2 & 0 & 1 & 0 & 1 & 1 \\
\hline 3 & 1 & 0 & 0 & 1 & 1 \\
\hline 4 & 1 & 0 & 0 & 1 & 1 \\
\hline 5 & 0 & 1 & 1 & 0 & 1 \\
\hline 6 & 1 & 1 & 1 & 0 & 1 \\
\hline 7 & 1 & 0 & 1 & 0 & 1 \\
\hline 8 & 1 & 0 & 1 & 1 & 1 \\
\hline 9 & 0 & 0 & 1 & 0 & 1 \\
\hline 10 & 0 & 0 & 1 & 1 & 1 \\
\hline 11 & 0 & 1 & 1 & 1 & 1 \\
\hline 12 & 1 & 1 & 1 & 1 & 1 \\
\hline
\end{tabular}


Результат вариантов сочетания, расположенный в строках (таблица 1 №п. п.), предусматривает 12 комбинаций признаков и отмечает фрагменты контура для совпадающего идентификатора группировки, для координат центров масс $X_{M J 1}, Y_{M J 1}$ фрагментов изображения J1, а также координат центров масс $X_{M J 2}$, $Y_{\text {MJ2 }}$ фрагментов изображения $\mathrm{J} 2$.

\section{ОБСУЖДЕНИЕ ПОЛУЧЕННЫХ РЕЗУЛЬТАТОВ}

Определение общей площади занимаемой дислокациями достигается использованием данных о принадлежности фрагментов ямкам травления согласно таблицы 1.

В случае отсутствия достоверного изображения J0 с данными о совпадении бинарных и основных значимых фрагментов множества $b=\left\{X_{m}, Y_{k}\right\} \in B$, когда не совпадают координаты фрагментов центров масс $X_{M J 2}, Y_{M J 2}$ изображения J2 и координат $X_{m J 0}$, $Y_{n J 0}$ изображения J0, могут использоваться сочетания других характерных признаков, указанных в таблице 1, № вариантов сочетания 2, 5, 9, 10, 11 .

Так или иначе, после получения признака идентификации (см. таблицу 1, столбец 5), результат в виде координат фрагментов, имеющих уникальный номер идентифицированной группировки фрагментов для конкретной ямки травления, будет указывать на координаты полигона ямки травления, которые будут затем использованы для расчета площади дислокации. Зная общую площадь всех ямок травления дислокаций и площадь эталона, возможен расчет количества дислокаций или занимаемой ими площади для пластины монокристалла GaAs.

\section{ВЫВОДЫ}

Комбинация признаков устраняет неоднозначность отображения ямок травления на поверхности пластины монокристалла полупроводника, освещаемой моноисточником МИИ-4. Таким образом, обнаружение контуров ямок травления дислокаций по матрице идентификации контура дислокации фрагментов ямки травления формирует основу для подсчета дислокаций. Учитывая возможности автоматизированного расчета площади ямок травления после идентификация, создана основа для построения информационной технологии определения плотности дислокаций для мониторинга качества пластин. Дальнейшие исследования предполагают совершенствование процедур расчета площадей дислокаций.

\section{REFERENCES}

[1] Kremnij monokristallicheskij v slitkah. Tehnicheskie usloviya : GOST 19658-81 [Dejstvitelen ot 01.01.83]. IPK Izdatelstvo standartov, 2001. p. 61.

[2] Standard Guide for Identification of Structures and Contaminants Seen on Specular Silicon Surfaces. ASTM Designation: F 154 00. Barr Harbor Drive, West Conshohockerv, 2000. p. 13, 128 Richards J. L. Etch Pits in Gallium Arsenide. J. L. Richards, A. J. Crocker. J. Appl. Phys. 1960. v. 31. R. 611.

[3] Senko, S.F., Zelenin, V.A. (2018). Ocenka razmerov topograficheskih defektov poluprovodnikovyh kremnievyh struktur [Estimation of the size of topographic defects of semiconductor silicon structures]. Pribory i metody izmerenij, vol. 9, no. 1, pp. 74-78.

[4] Pritchin, S. (2016). Razrabotka tehnologii proizvodstva podlozhek arsenida galliya dlya izdelij mikroelektroniki (doktorskaya rabota). Retrieved from: https://nure.ua/wp-content/uploads/2018/Dissertation/diss_Pritchin.pdf.

[5] Samojlov, A., Shevchenko, I. (2016). Razrabotka metodov vydeleniya binarizovannyh fragmentov yamok travleniya plastiny poluprovodnika [Development of methods for isolating binarized fragments of etching pits of a semiconductor wafer]. Tehnologicheskij audit i rezervy proizvodstva, vol. 29 (68), no. 3/1 (29), part 2, pp. 60-68.

[6] Samojlov, A., Shevchenko, I. (2010). Rezultati analizu adaptivnoyi obrobki yarkisnih porogiv fragmentiv cifrovogo rastrovogo zobrazhennya plastini napivprovidnika [The results of the analysis of the adaptive processing of bright thresholds of fragments of digital raster image of a semiconductor wafer]. Vcheni zapiski Tavrijskogo nacionalnogo universitetu imeni V.I. Vernadskogo, vol. 29 (68), no. 5, chastina 2, pp. 51-55.

[7] Gonzales, R., Woods, R. (2005). Digital Image Processing. Addison-Wesley Publishing Company.

[8] Samoilov, A., Shevchenko, I. (2016). Grouping method of image fragments of adjacent dislocation etch pits of the semiconductor wafer [Grouping method of image fragments of adjacent dislocation etch pits of the semiconductor wafer] EUREKA: Physics and Engineering, no. 4 (5), pp. 47-54.

\section{СПИСОК ИСПОЛЬЗОВАННОЙ ЛИТЕРАТУРЫ}

[1] Кремний монокристаллический в слитках. Технические условия: ГОСТ 19658-81 [Действителен от 01.01.83]. ИПК Издательство стандартов. (2001). 61 с.

[2] Richards, J. L., Crocker, A. J. (2000). Standard Guide for Identification of Structures and Contaminants Seen on Specular Silicon Surfaces. ASTM Designation: F 154 - 00. Barr Harbor Drive, West Conshohockerv. p. 13, 128-Etch Pits in Gallium Arsenide. J. Appl. Phys., v. 31, p. 611.

[3] Сенько, С. Ф., Зеленин, В. А. (2018). Оценка размеров топографических дефектов полупроводниковых кремниевых структур. Приборы и методы измерений. Т. 9, № 1, 74-84.

[4] Притчин, С. (2016). Разработка технологии производства подложек арсенида галлия для изделий микроэлектроники (докторская работа). URL: https://nure.ua/wp-content/uploads/2018/Dissertation/diss_Pritchin.pdf. 
[5] Самойлов, А. Н., Шевченко, И. В. (2016). Разработка методов выделения бинаризованных фрагментов ямок травления пластины полупроводника. Технологический аудит и резервы производства, 2016. 3/1 (29), 60-68.

[6] Самойлов, А. М., Шевченко, І. В. (2018). Результати аналізу адаптивної обробки якісних порогів фрагментів цифрового растрового зображення пластини напівпровідника. Вчені записки Таврійського національного університету імені В. I. Вернадського. Том 29 (68), № 5, частина 2, 51-55.

[7] Gonzales, R. and Woods, R. (2005). Digital Image Processing. Addison-Wesley Publishing Company, 2005. p. 976.

[8] Samoilov, A., Shevchenko, I. (2016). Grouping method of image fragments of adjacent dislocation etch pits of the semiconductor wafer. EUREKA : Physics and Engineering, no. 2016. 4 (5), pp. 47-54. 